\section{A) Check for updates}

Cite this: Nanoscale, 2020, 12, 18938

\title{
Janus nanoparticles designed for extended cell surface attachment $\dagger$
}

\author{
Reshma Kadam, ${ }^{a}$ Jaee Ghawali, ${ }^{a}$ Mario Waespy, ${ }^{\mathrm{b}}$ Michael Maas (DD *a,c and \\ Kurosch Rezwan ${ }^{a, c}$
}

\begin{abstract}
In this study, we present Janus nanoparticles that are designed for attaching to a eukaryotic cell surface with minimal cell uptake. This contrasts the rapid uptake via various endocytosis pathways that non-passivated isotropic particles usually encounter. Firmly attaching nanoparticles onto cell surfaces for extended periods of time can be a powerful new strategy to employ functional properties of nanoparticles for noninvasive interrogation and manipulation of biological systems. To this end, we synthesized rhodaminedoped silica $\left(\mathrm{SiO}_{2}\right)$ nanoparticles functionalized with 1,2-distearoyl-sn-glycero-3-phosphoethanolamine (DSPE) on one hemisphere of the nanoparticle surface and high-molecular-weight long-chain poly(ethylene glycol) on the other one using the wax-Pickering emulsion technique. Nanoparticle localization was studied with NIH 3 T3 rat fibroblasts in vitro. In these studies, the Janus nanoparticles adhered to the cell surface and, in contrast to isotropic control particles, only negligible uptake into the cells was observed, even after $24 \mathrm{~h}$ of incubation. In order to characterize the potential endocytosis pathway involved in the uptake of the Janus nanoparticles in more detail, fibroblasts and nanoparticles were incubated in the presence or absence of different endocytosis inhibitors. Our findings indicate that the Janus particles are not affected by caveolae- and receptor-mediated endocytosis and the prolonged attachment of the Janus nanoparticles is most likely the result of an incomplete macropinocytosis process. Consequently, by design, these Janus nanoparticles have the potential to firmly anchor onto cell surfaces for extended periods of time and might be utilized in various biotechnological and biomedical applications like cell surface tagging, magnetic manipulation of the cell membrane or non-invasive drug and gene delivery.
\end{abstract}

\author{
Received 27th May 2020, \\ Accepted 12th August 2020 \\ DOI: $10.1039 / \mathrm{d} 0 \mathrm{nr} 04061 \mathrm{~d}$ \\ rsc.li/nanoscale
}

\section{Introduction}

Based on the powerful properties of nanoparticles in biomedical applications, it is highly desirable to attach nanoparticles to the plasma membrane of living cells. However, nanoparticles that adhere to the surface of eukaryotic cells, in general, are rapidly incorporated via various endocytosis pathways, depending on physical and chemical parameters of the particles including size, shape, charge and surface chemistry. $^{1-3}$ This process, which usually leads to nanoparticles being engulfed in endosomes and transported into the cytosol, makes it extremely difficult to retain nanoparticles at the cell surface for relevant periods of time.

\footnotetext{
${ }^{a}$ Advanced Ceramics, University of Bremen, Am Biologischen Garten 2, 28359 Bremen, Germany.E-mail:michael.maas@uni-bremen.de

${ }^{b}$ Center for Biomolecular Interactions Bremen, Faculty 2 (Biology/Chemistry), University of Bremen, Leobener Straße 5, NW2 28359 Bremen, Germany ${ }^{c}$ MAPEX Centre of Materials and Processes, University of Bremen, 28359 Bremen, Germany

$\dagger$ Electronic supplementary information (ESI) available: Supplementary figures. See DOI: 10.1039/d0nr04061d
}

Nanoparticle uptake into cells can be mostly avoided by coating the particles with a passivation layer, which is commonly comprised of poly(ethylene glycol) (PEG). ${ }^{4,5}$ This socalled stealth coating prevents interactions with serum proteins and cell surface receptors while also reducing nonspecific physico-chemical interactions between particles and cell surface molecules. On the other hand, nanoparticles can be modified with a wide variety of surface functionalizations ${ }^{6}$ to tailor the particles for specific interactions including antigen-antibody binding, receptor-mediated recognition or anchoring of lipids into the plasma membrane. ${ }^{1}$ This results in two opposing functionalization strategies: minimization of nanoparticle-cell interactions altogether via stealth coating or utilizing specific interactions that quickly result in endocytosis. The fundamental approach of the present work is to use Janus nanoparticles to incorporate both functionalization strategies on opposing hemispheres of the same nanoparticle to create particles that firmly adhere to cell surfaces without being promptly endocytosed. ${ }^{5,7}$

Janus particles, named after the Roman god with faces directed towards the past and the future, have gained popularity since their introduction by de Gennes in $1991^{8}$ and have 
since been used in various applications like bioimaging, ${ }^{9}$ drug delivery systems, ${ }^{10-14}$ magnetotherapy ${ }^{15}$ or bacterial extraction. ${ }^{16,17}$ Janus particle preparation can be achieved by several methods including masking at interfaces, ${ }^{18}$ templating using emulsions ${ }^{19}$ and self-assembly. ${ }^{20}$ While most methods result in a relatively small number of produced Janus particles, Janus particle preparation using the wax Pickering emulsion method is a scalable method which easily yields gram quantities of approximately half-coated particles. In this approach, one hemisphere of the particle is masked at the surface of solidified wax emulsion droplets and hence the exposed particle hemisphere can be modified with a separate functionality. ${ }^{21-23}$

In a seminal paper from $2013^{7}$ and its follow-up from $2015,{ }^{24}$ Gao and $\mathrm{Yu}$ explored the endocytosis and fate of micron-sized Janus particles which were coated with anti-CD3 antibodies and a passivation layer of bovine serum albumin (BSA) on respective sides. In this first detailed study on the uptake of Janus particles, the authors were able to show a twostep endocytosis pathway in which the antibody-coated side was progressively engulfed via receptor-mediated endocytosis. Afterwards the BSA-coated side was covered with a further extension of the cell membrane in an actin-mediated process. Full endocytosis of these Janus microparticles by Jurkat $\mathrm{T}$ cells was reported to take 1-5 min, which was significantly longer than endocytosis of isotropic particles. In a similar approach, Sanchez et al. studied the effect of PEG functionalization on one hemisphere of Janus microparticles on their uptake by macrophages. The passivation with PEG instead of BSA resulted in strongly reduced internalization of about $25 \%$ of the incubated particles measured after $30 \mathrm{~min}$. With anti-CD3 antibodies on the binding hemisphere, they report that also about $25 \%$ of the particles remain bound to the cell surface after an incubation time of $30 \mathrm{~min} .{ }^{5}$ Note that the reported experimental studies have been carried out with micron-sized particles whose anisotropic features are easily visualized with fluorescence microscopy. Compared to microparticles, nanoparticles interact significantly different with biological systems due to their much higher surface area to volume ratio and dimensions that are much closer to proteins which might lead to drastically different uptake behavior. ${ }^{25}$ Additionally, a fairly large body of computational studies about Janus particle interactions with phospholipid membranes provide theoretical background to some aspects of these observations which are summarized in a recent review. ${ }^{26}$

Here, we will build on the findings for microscale Janus particles and introduce a nanoscale Janus particle design, which features membrane anchoring phospholipids on one hemisphere and a PEG stealth coating on the other. By creating such dual-functionalized particles, we aim to anchor the nanoparticles onto eukaryotic cell membranes and at the same time to inhibit the endocytosis process that leads to uptake of these particles into cells via the passivation layer on the other half of the particle surface. To this end, we synthesized dyedoped silica nanoparticles (50 $\mathrm{nm}$ diameter) functionalized with 1,2-distearoyl-sn-glycero-3-phosphoethanolamine (DSPE) on one side and PEG on the other side of the particle surface.
To facilitate insertion of the phospholipid DSPE into the cell membrane, it was connected to the particle surface via a flexible PEG-linker. The particles were first characterized in terms of their material properties and colloidal behavior. In in vitro cell culture studies, the Janus nanoparticles were analyzed jointly with their isotropic (non-Janus) pendants as controls in a mouse fibroblasts cell culture model using NIH 3T3 cells regarding their association to the plasma membrane and their endocytosis properties. Cellular location and distribution patterns of the particles were determined by confocal laser scanning microscopy (CLSM) coupled with Z-stack imaging and transmission electron microscopy (TEM). Furthermore, we incubated the nanoparticles together with NIH 3T3 cells in the presence or absence of various endocytose inhibitors to reveal associated internalization pathways.

\section{Materials and methods}

\section{Materials}

For Janus nanoparticle synthesis and characterization, L-arginine (product no. A-5006), tetraethyl orthosilicate (TEOS, product no. 78-10-4), Rhodamine B Isothiocyanate (RBITC, mixed isomers, product no. MKBJ9031 V), 3-aminopropyl triethoxysilane (APTES; product no. 440140), sodium dodecyl sulphate (SDS, product no. 436143), phosphate buffered saline (PBS, product no. P4417), ninhydrin reagent (product no. 151173), lysozyme from chicken egg white $\left(70000 \mathrm{U} \mathrm{mg}^{-1}\right.$, product no. 62971), $N$-(3-dimethylaminopropyl)- $N^{\prime}$-ethylcarbodiimide hydrochloride (EDC, product no. E6383), $N$-hydoxysuccinimide (NHS, product no. 130672), and absolute ethanol (>98\%, lot no. SZBB1570 V) were all purchased from Sigma Aldrich (Darmstadt, Germany). Albumin from bovine serum fluorescein isothiocyanate conjugate (FITC-BSA, product no. A23015) from ThermoFischer Scientfic (Schwerte, Germany), paraffin wax (melting point $75{ }^{\circ} \mathrm{C}$ to $90^{\circ} \mathrm{C}$, product no. 8002-74-2) and toluene (product no. 1083251000) from Merck Millipore (Darmstadt, Germany), 2-[methoxypoly(ethyleneoxy)propyl]trimethoxy-silane (mPEG silane, molecular weight $5000 \mathrm{~g} \mathrm{~mol}^{-1}$, product no. MPEG-SIL-5000) from Laysan Bio. Inc. (Arab, AL, USA) and DSPE-PEG(2000)-carboxylic acid (DSPE-PEG-COOH, product no. 880135) was purchased from Avanti Polar Lipids, Inc. (Alabaster, Alabama, USA). All of the chemicals were used as-received and without any further purification.

In vitro cell culture experiments were carried out using the mouse fibroblast cell line NIH 3T3 (passage number 8-25, product no. 400101), which was purchased from CLS Cell Lines Services GmbH (Eppelheim, Germany) and cultured in Dulbecco's Vogt modified Eagle's minimum essential medium (DMEM; high glucose, lot no. 1206393) supplemented with fetal calf serum (FCS, product no. 010M3395) and antibioticantimycotic solution (AB/AM, product no. 1209917) were purchased from Invitrogen (Germany). Trypsin-ethylenediaminetetraacetic acid $(0.25 \%$ trypsin, $0.02 \%$ EDTA, product no. SLBG4376), Triton X-100 (product no. MKBL5839 V), phos- 
phate buffered saline (PBS, product no. SLBF5741 V), paraformaldehyde (PFA, product no. 53260), osmium tetroxide solution $\left(\mathrm{OsO}_{4}, 4 \%\right.$ in water, product no. 75632), glutaraldehyde solution (Grade, 1.25\% in water, product no. G5882), 4',6-diamidino-2-phenylindole (DAPI, $0.5 \mu \mathrm{g} \mathrm{ml} \mathrm{m}^{-1}$, product no. 1242642), Alexa fluor 488 phalloidin (AF488, $2 \mathrm{U} \mathrm{ml}^{-1}$, product no. 1151587), wortmannin (lot no. 023M4072), nystatin (lot no. 020M13491), sucrose (lot no. 1076511000), agar (lot no. A1296) and an epoxy embedding medium kit (Epon 812 substitute, product no. 45359) were purchased from Sigma Aldrich (Germany). Cell toxicity was measured using water-soluble tetrazolium salt assay (WST-1, Roche Diagnostics $\mathrm{GmbH}$, Germany, product no. 14310100) and lactate dehydrogenase assay (LDH), Pierce assay (Thermo Scientific, Germany, lot no. OL17881450).

\section{Methods}

For describing the step-wise surface functionalizations introduced on the Janus particles, we use the forward slash (/) to denote the segregated sides on a single particle surface. For example, "PEG/DSPE" describes a RBITC-doped silica particle that has been functionalized on one side with PEG and on the other side with DSPE (the PEG linker between DSPE and the particle surface as well as the APTES-functionalization to attach this PEG linker have been omitted for brevity).

\section{Preparation of $\mathrm{NH}_{2}$-functionalized RBITC-doped $\mathrm{SiO}_{2}$ Janus nanoparticles}

RBITC-doped $\mathrm{SiO}_{2}$ nanoparticles were prepared following the protocol described by Bollhorst et $a l^{27}$ which was adapted from the method previously established by Watanabe et $a .^{28}$ The $50 \mathrm{~nm}$ RBITC doped $\mathrm{SiO}_{2}$ nanoparticles were functionalized with $\mathrm{NH}_{2}$ groups using the silane reagent APTES. The presence of amino groups on the silica nanoparticle surface is important for further functionalization with DSPE. The number of $\mathrm{NH}_{2}$ groups for surface functionalization was calculated to cover half the area of the nanoparticle surface. $10 \mathrm{mg}$ $\mathrm{ml}^{-1}$ of rhodamine-doped silica nanoparticles were suspended in $20 \mathrm{ml}$ toluene using a sonication bath for $10 \mathrm{~min}$ and subjected to heating at $70^{\circ} \mathrm{C} .21 \mu \mathrm{l}$ APTES was added dropwise to the solution and stirred for $6 \mathrm{~h}$. After functionalization, nanoparticles were washed twice with both ethanol and water sequentially. The nanoparticles were air-dried and stored at room temperature.

The density of the grafted $\mathrm{NH}_{2}$ groups on $\mathrm{NH}_{2}$-functionalized RBITC-doped $\mathrm{SiO}_{2}$ was then quantified spectrophotometrically using the reagent ninhydrin by adapting the procedure described by Soto-Cantu et al. ${ }^{29}$ The nanoparticles were dispersed in absolute ethanol at a concentration of $10 \mathrm{mg} \mathrm{ml}^{-1}$ using ultrasonication for $10 \mathrm{~min} .200 \mu \mathrm{l}$ of $0.35 \%(\mathrm{w} / \mathrm{v})$ solution of ninhydrin in ethanol was added to the particle solution followed by ultrasonication for $5 \mathrm{~min}$. This sonicated ninhydrin-nanoparticle mixture was incubated in a heating bath at $65^{\circ} \mathrm{C}$ for $5 \mathrm{~min}$, which was further left to cool for $5 \mathrm{~min}$. The Eppendorf tubes were centrifuged to remove any unreacted ninhydrin molecules at $13000 \mathrm{rpm}$ for $20 \mathrm{~min}$. The absor- bance of $1 \mathrm{ml}$ of the supernatant was then measured using a Multiscan Go spectrometer at $588 \mathrm{~nm}$. The amount of unbound ninhydrin was used to calculate the amount of bound ninhydrin, from which the amount of $\mathrm{NH}_{2}$ groups per $\mathrm{nm}^{2}$ of the surface area of the nanoparticles was deducted. Increasing concentrations of a standard solution of hexylamine ( $0.12 \mathrm{mM}$ to $0.87 \mathrm{mM}$ ) was used to prepare a calibration curve. All particle types were measured in triplicates and the amount of amine functional groups per $\mathrm{nm}^{2}$ were expressed along with standard deviations.

\section{Preparation of wax Pickering emulsion droplets}

According to the procedure we described previously, ${ }^{16,23}$ which is based on the method established by Granick et al., ${ }^{21}$ a Pickering emulsion was prepared with molten wax droplets and the $\mathrm{NH}_{2}$-functionalized RBITC-doped $\mathrm{SiO}_{2}$ nanoparticles using SDS as the surfactant. The solidified wax-Pickering emulsion droplets were washed several times with water using vacuum filtration and stored in the dark.

\section{Preparation of PEG/DSPE functionalized $\mathrm{SiO}_{2}$ Janus nanoparticles}

The wax-embedded particles were functionalized first with PEG on the exposed hemispheres. To this end, $10 \mathrm{mg}$ of wax droplets were incubated with $20 \mathrm{mg}$ of mPEG silane using the protocol mentioned in our previous work. ${ }^{16}$ SEM (Supra40, Zeiss, Germany) with an acceleration voltage of $15 \mathrm{kV}$ was used to confirm the morphology of the wax colloidosomes before and after functionalization. For the PEG-functionalization step, twice the calculated monolayer concentration of the silane was used in the functionalization protocol. The nanoparticles were then extracted by dissolving the wax spheres in cyclohexane. The as-prepared $\mathrm{NH}_{2}$ /PEG functionalized Janus nanoparticles were air-dried and stored in the dark. Following the protocol of carbodiimide based coupling described by the manufacturer, ${ }^{30,31} 0.5 \mathrm{ml}$ of $10 \mathrm{mg} \mathrm{ml}^{-1} \mathrm{NH}_{2} /$ PEG functionalized Janus nanoparticles were incubated with $0.5 \mathrm{ml}$ of $10 \mathrm{mg}$ $\mathrm{ml}^{-1}$ of DSPE-PEG-COOH in $4 \%(\mathrm{v} / \mathrm{v})$ ethanol-water to obtain lipid-functionalized Janus nanoparticles. To this mixture, EDC and NHS were added at molar ratio of $5: 1$, also prepared in $4 \%(\mathrm{v} / \mathrm{v})$ ethanol-water and thoroughly vortexed for $1 \mathrm{~min}$. The reaction mixture was incubated at $4{ }^{\circ} \mathrm{C}$ under constant shaking. After $16 \mathrm{~h}$, the obtained DSPE/PEG RBITC doped $\mathrm{SiO}_{2}$ Janus nanoparticles were washed several times, dried and stored in the dark.

With the same approach, further nanoparticles have been prepared with and without Janus functionalization for control experiments: isotropic unfunctionalized particles, fully APTEScoated particles, fully PEG-coated particles, fully DSPE-coated particles and anisotropic APTES/PEG Janus nanoparticles. Isotropic unfunctionalized particles were utilized as prepared. The preparation of the fully APTES-coated particles is mentioned above. For the preparation of fully PEG-coated particles, $5 \mathrm{ml}$ of $10 \mathrm{mg} \mathrm{ml}{ }^{-1}$ RBITC-doped $\mathrm{SiO}_{2}$ nanoparticles in ethanol was incubated with $40 \mu$ l of mPEG silane incubated for $8 \mathrm{~h}$ under stirring at room temperature. ${ }^{32}$ The silanized 
particles were washed several times using absolute ethanol and $\mathrm{ddH}_{2} \mathrm{O}$ using alternative centrifugation and redispersion cycles. In case of the fully DSPE-coated particles, $5 \mathrm{ml}$ of $10 \mathrm{mg} \mathrm{ml}^{-1}$ fully APTES-coated particles in ethanol were incubated with $40 \mu \mathrm{l}$ of DSPE-PEG-COOH using the EDC-NHS coupling procedure.

\section{Particle characterization}

The various synthesized nanoparticles were visualized using transmission electron microscopy (TEM, EM 900, Zeiss, Germany). For sample preparation, $4 \mu$ l of nanoparticle dispersion were pipetted on carbon-coated copper grids (Plano GmbH, Wetzlar, Germany) and analyzed using TEM which was operated at $80 \mathrm{kV}$. The size and zeta potential characterization of the nanoparticles after each functionalization step was performed using a Zeta Sizer ZSP (Malvern instruments, USA). The mean values of the measurements were obtained from three independently prepared replicates.

PEG functionalization of the nanoparticles was assessed by measuring the adsorption of FITC-BSA. $10 \mathrm{mg}$ nanoparticles were dispersed in $1 \mathrm{mM}$ PBS and incubated with $2 \mu \mathrm{mol}$ of FITC-BSA per gram of nanoparticles for $1 \mathrm{~h}$ at room temperature. This mixture was then washed thrice with ethanol-water solution to remove unbound FITC-BSA. The residual and adsorbed FITC-BSA concentrations were then quantified using a fluorescence plate reader (Chameleon V, Hidex, Germany). By measuring the fluorescence signal at $\lambda_{\mathrm{ex}}: 495 \mathrm{~nm}, \lambda_{\mathrm{em}}: 525 \mathrm{~nm}$ of known increasing concentrations of FITC-BSA (1 nmol to $20 \mathrm{nmol}$ FITC $\mathrm{ml}^{-1}$ of PBS), a calibration curve was prepared, which was used to calculate unknown FITC-BSA concentrations of the samples.

\section{Exposure of NIH 3T3 fibroblasts to Janus nanoparticles}

The cells were cultured according to the procedure described by Shahabi et al. and Holthaus et al. ${ }^{32,34}$ DMEM supplemented with $10 \%$ FCS and $1 \% \mathrm{AB} / \mathrm{AM}$ were used as the medium for culturing NIH 3T3 cells in an incubator (C200, Labortechnik Göttingen, Germany) at $37{ }^{\circ} \mathrm{C}, 10 \% \mathrm{CO}_{2}$ and $95 \%$ relative humidity (RH). The cells were cultured in $75 \mathrm{~cm}^{2}$ flasks for one week and the culture medium was changed every second day. Cells were then trypsinized using trypsin-EDTA solution, counted using the Scepter ${ }^{\mathrm{TM}} 2.0$ handheld cell counter (Millipore, Germany) and diluted to attain a final cell density of $1 \times 10^{4}$ cells per $\mathrm{ml}$ of culture medium. $1 \mathrm{ml}$ of cell suspension was deposited onto $15 \mathrm{~mm}$ glass coverslips (lot no. 0983, VWR, Germany) placed in each well of a 6-well polystyrene cell culture plate (NUNC, Fischer Scientific, Germany) and incubated at $37{ }^{\circ} \mathrm{C}, 10 \% \mathrm{CO}_{2}$ and $95 \% \mathrm{RH}$. After $30 \mathrm{~min}$ of incubation of the cells on the coverslips, $100 \mu \mathrm{l}$ of the respective nanoparticle dispersion ( $1 \mathrm{mg}$ of $\mathrm{NPs} / \mathrm{ml}$ of DMEM + FCS $(10 \%)+\mathrm{AB} / \mathrm{AM}(1 \%)$ were added onto the cells and incubated for $1 \mathrm{~h}, 6 \mathrm{~h}$ and $24 \mathrm{~h}$ at $37^{\circ} \mathrm{C}$ covered with aluminium foil and under shaking conditions. NIH 3 T3 cells were also incubated in the absence of nanoparticles as a control.

\section{Cell staining and imaging}

After exposure of the cells to the nanoparticles for the respective time points, the nanoparticle dispersions were removed and cells were washed thrice with pre-warmed $\left(37^{\circ} \mathrm{C}\right) \mathrm{PBS}$ to remove any loosely bound (for example as a result of sedimentation) or non-internalized nanoparticles. The cells were then fixed with 4\% paraformaldehyde (PFA) for $15 \mathrm{~min}$ at RT and then permeabilized using $0.5 \%$ Triton X-100 for $3 \mathrm{~min}$ at RT. The cells were then fluorescently stained with $1 \mathrm{ml}$ of staining solution which contained DAPI $\left(0.5 \mu \mathrm{g} \mathrm{m} \mathrm{m}^{-1}\right.$ of $1 \mathrm{mM}$ PBS $)$ for the cell nuclei and phalloidin AF488 $\left(10 \mu \mathrm{g} \mathrm{ml} \mathrm{m}^{-1}\right.$ of $1 \mathrm{mM}$ PBS) for the cytoskeleton. Nanoparticle uptake was analyzed using an AX-10 fluorescence microscope (Zeiss, Germany). To visualize NIH 3T3 cells and the attachment of the nanoparticles, three fluorescence channels were used: FS02 for DAPI $\left(\lambda_{\mathrm{ex}}\right.$ : 300-400 nm, $\left.\lambda_{\mathrm{em}}:>450 \mathrm{~nm}\right)$, FS09 for AF488 $\left(\lambda_{\mathrm{ex}}: 450-490 \mathrm{~nm}\right.$, $\left.\lambda_{\mathrm{em}}:>515 \mathrm{~nm}\right)$ and $\operatorname{FS} 15\left(\lambda_{\mathrm{ex}}: 530-558 \mathrm{~nm}, \lambda_{\mathrm{em}}:>590 \mathrm{~nm}\right)$. CLSM Images were acquired using a Zeiss LSM 880 (Zeiss, Jena, Germany) with a Plan-Apochromat 63/1.4 DIC M27 lens. The following settings were used for measurement: DAPI: track 2 Ch1 emission filter $410-494 \mathrm{~nm}$, excitation at $405 \mathrm{~nm}$ (exposure time $20 \mathrm{~ms}$ ). Actin AF488 phalloidin: track $1 \mathrm{ChS} 1$ emission filter $490-544 \mathrm{~nm}$, excitation at $488 \mathrm{~nm}$ (exposure time $40 \mathrm{~ms}$ ). RBITC nanoparticles: track $2 \mathrm{Ch} 2$ emission filter 566-685 nm, excitation at $561 \mathrm{~nm}$ (exposure time $50 \mathrm{~ms}$ ). The pinhole setting for all the measurements was $50 \mu \mathrm{m}$ for the 3 channels mentioned above.

\section{Localization of nanoparticles using transmission electron microscopy}

Transmission electron microscopy (TEM) was performed following the procedure described by Apte et al. ${ }^{35}$ NIH 3T3 cells incubated for $1 \mathrm{~h}, 6 \mathrm{~h}$ and $24 \mathrm{~h}$ in the absence or presence of nanoparticles, respectively, were washed twice with $1 \mathrm{mM}$ PBS followed by trypsin-EDTA treatment to detach cells. Cells were centrifuged at $6000 \mathrm{rpm}$ for $3 \mathrm{~min}$ and further treated for $1 \mathrm{~h}$ with $2.5 \%$ formaldehyde in $0.1 \mathrm{M} \mathrm{Na}$-cacodylate at room temperature. After that, cells were washed with PBS and stained using $1 \mathrm{mM}$ osmium tetroxide solution in PBS for $1 \mathrm{~h}$ at RT, resulting in a black cell pellet. This cell pellet was further washed twice with PBS, embedded in $2 \%$ agar in water and solidified at $4{ }^{\circ} \mathrm{C}$. Small $1 \mathrm{~mm}$ wide blocks were cut into squares and dehydrated with increasing concentrations of ethanol such as 30\% (twice for $5 \mathrm{~min}$ each), 50\% (twice for $5 \mathrm{~min}$ each), 70\% (3 times for $10 \mathrm{~min}$ each), 90\% (3 times for $10 \mathrm{~min}$ each), and 100\% (twice for $15 \mathrm{~min}$ each). Epoxy resin (Epon 812) embedding was performed on the dehydrated agar blocks containing the fixed cell pellet followed by cutting of ultrathin sections, using an ultramicrotome (Ultracut R, Leica, Wetzlar, Germany).

\section{Inhibition of endocytosis}

Two different commercial endocytosis inhibitors were used to characterize the potential uptake pathways that are triggered by our synthetic nanoparticles. NIH 3T3 cells were incubated 
in the presence of hypertonic medium containing highly concentrated sucrose $(0.45 \mathrm{M})$, nystatin $(10 \mu \mathrm{M})$ or wortmannin (300 $\mathrm{nM}$ ) in combination with the nanoparticles for $1 \mathrm{~h}, 6 \mathrm{~h}$ or $24 \mathrm{~h}$, respectively. To prevent effective endocytosis, cells were incubated at $4{ }^{\circ} \mathrm{C}$ for $1 \mathrm{~h}, 6 \mathrm{~h}$ and $24 \mathrm{~h}$ in the absence or presence of nanoparticles. Following incubation, cells were fluorescently stained and imaged as described above. As control, cells were analyzed in the absence of endocytosis inhibitors at all the respective time points.

\section{Determination of cell viability}

In order to determine whether our synthetic nanoparticles and/or the endocytosis inhibitors affected cell viability, a colorimetric water-soluble tetrazolium salt (WST-1) cell proliferation assay was performed as described in ref. 33 and 36. In brief, $100 \mu$ l of WST-1 cell proliferation reagent was added to the cell culture wells and incubated for $1 \mathrm{~h}$ at $37{ }^{\circ} \mathrm{C}, 10 \% \mathrm{CO}_{2}$ and $95 \% \mathrm{RH}$. The medium containing the WST-1 proliferation reagent was then collected from the wells and centrifuged at $10000 \mathrm{~g}$ for $5 \mathrm{~min}$ to remove the nanoparticles. The cells attached to the bottom of the cell culture plates were trypsinized using trypsin-EDTA solution and centrifuged at $1000 \mathrm{~g}$ to collect the cells. Formazan, which is a product of the enzymatic conversion of tetrazolium within living cells, was quantified by measuring adsorption at $450 \mathrm{~nm}$ using a multiscan GO spectrophotometer (Thermo Scientific, Finland). DMEM + FCS (10\%) + $\mathrm{AB} / \mathrm{AM}(1 \%)$ which has not been in contact with cells was incubated with the WST-1 reagent was used as a background signal control.

Intra- and extracellular quantities of lactate dehydrogenase $(\mathrm{LDH})$ were quantified in the absence and presence of the nanoparticle dispersions using the Pierce assay. ${ }^{33,36}$ For the quantification of extracellular LDH released by damaged cells due to the loss of membrane integrity, the media was collected after incubation of the cells with the nanoparticles and centrifuged at $10000 \mathrm{~g}$ for $3 \mathrm{~min}$ to remove any dispersed nanoparticles. The quantification of released $\mathrm{LDH}$ was performed following supplier instructions using $50 \mu \mathrm{L}$ of cell culture medium. To obtain the intracellular LDH concentration, cells were detached from cell culture plates by trypsin-EDTA treatment and centrifuged at $600 \mathrm{~g}$ for $10 \mathrm{~min}$ to harvest the cells. Afterwards, cell lysis was performed using lysis solution (1\% $\mathrm{v} / \mathrm{v}$ Triton $\mathrm{X}-100$ in $0.9 \% \mathrm{w} / \mathrm{v} \mathrm{NaCl}$ in water). The suspension was mixed by pipetting several times to obtain a clear solution and $50 \mu \mathrm{l}$ of the cell lysate was used for the LDH assay. After incubation for $30 \mathrm{~min}$ in the dark, the absorbance of the samples after addition of $50 \mu \mathrm{l}$ of the assay solution was analyzed at $490 \mathrm{~nm}$ along with a reference measurement at $680 \mathrm{~nm}$ using a Multiscan GO spectrophotometer. Cells incubated in DMEM + FCS (10\%) and AB/AM (1\%) were used as minimum LDH activity control (100\% cell viability) and cells incubated with $2 \% \mathrm{v} / \mathrm{v}$ Triton X-100 in $1 \mathrm{mM}$ PBS buffer were used as maximum LDH activity control ( $0 \%$ cell viability). The amount of $\mathrm{LDH}$ was normalized to the total $\mathrm{LDH}$ concentration (extracellular LDH + intracellular LDH).

\section{Results and discussion}

\section{Particle characterization}

Synthesis and functionalization of DSPE/PEG Janus nanoparticles with RBITC-doped $\mathrm{SiO}_{2}$ cores is based on our prior protocol with only minor adaptations (Fig. 1). ${ }^{16,23}$ Briefly, RBITC-doped $\mathrm{SiO}_{2}$ nanoparticles are functionalized with APTES to provide $\mathrm{NH}_{2}$-groups on the particle surface. These particles are added to a heated wax-in-water emulsion to generate wax Pickering emulsion droplets decorated with the $\mathrm{NH}_{2}$ functionalized $\mathrm{SiO}_{2}$ nanoparticles. Here, the surfactant SDS is used to hydrophobize the particle surfaces. SEM images of the wax Pickering emulsion droplets prepared using APTES-functionalized nanoparticles are shown in Fig. S1 in the ESI. $\dagger$ The size of the solidified wax Pickering emulsion droplets decorated with amino-functionalized nanoparticles ranged from $30 \mu \mathrm{m}$ to $80 \mu \mathrm{m}$ in diameter (Fig. S1A $\dagger$ ), with partially embedded nanoparticles on the surfaces of the wax spheres (Fig. S1B $\dagger$ ). The particles mostly remained on the surface of wax even after several washing steps as observed in our previous work ${ }^{16,23}$ thus providing large-scale preparation of Janus nanoparticles.

After the cooling and washing steps, the solvent-exposed particle hemispheres on the solidified wax droplets are functionalized with PEG-silane. Afterwards, the wax is dissolved and the particles are thereby released from the wax spheres. The originally masked $\mathrm{NH}_{2}$ groups are then functionalized with COOH-PEG-DSPE via EDC-NHS chemistry. Additionally, further isotropic and Janus nanoparticles were prepared as listed in Table 1 as controls for the in vitro cell culture studies.

The prepared particles were characterized regarding their size and zeta potential by dynamic light scattering (DLS) before they were used for cell experiments. In order to assess the behavior of the particle dispersion during the cell culture experiments, the particles were analyzed both as a dispersion in a $4 \mathrm{vol} \%$ ethanol/water mixture and dispersed in the cell culture medium (DMEM + FCS (10\%) + AB/AM (1\%)) which was later used in the cell culture studies. DLS analysis of the unfunctionalized RBITC $\mathrm{SiO}_{2}$ nanoparticles dispersed in the ethanol/water mixture revealed a size of $50.5 \pm 6 \mathrm{~nm}$ (Table 1). A similar hydrodynamic diameter of $52.4 \pm 7 \mathrm{~nm}$ (Table 1) was recorded when these nanoparticles were dispersed in the cell culture medium, showing that colloidal stability was maintained under these conditions. However, APTES-functionalization of the RBITC $\mathrm{SiO}_{2}$ nanoparticles resulted in a moderate increase of the particle sizes in cell culture medium (62.4 \pm $4 \mathrm{~nm}$ ) indicating slightly decreased colloidal stability, possibly as a result of protein adsorption (mainly BSA as the main constituent of FCS) on the positively charged nanoparticle surface. $^{37}$ Isotropic PEG functionalization resulted in nanoparticles with a hydrodynamic diameter of $60.4 \pm 10.8 \mathrm{~nm}$ measured in ethanol/water (Table 1). In addition, DLS analysis of PEG-functionalized nanoparticles in cell culture medium lead to a similar hydrodynamic diameter of $58.7 \pm 2.6 \mathrm{~nm}$, most likely due to the suppressed protein adsorption of the PEGylated particle surface. ${ }^{33}$ The slight increase in hydrodyn- 


\section{Pickering emulsion preparation}

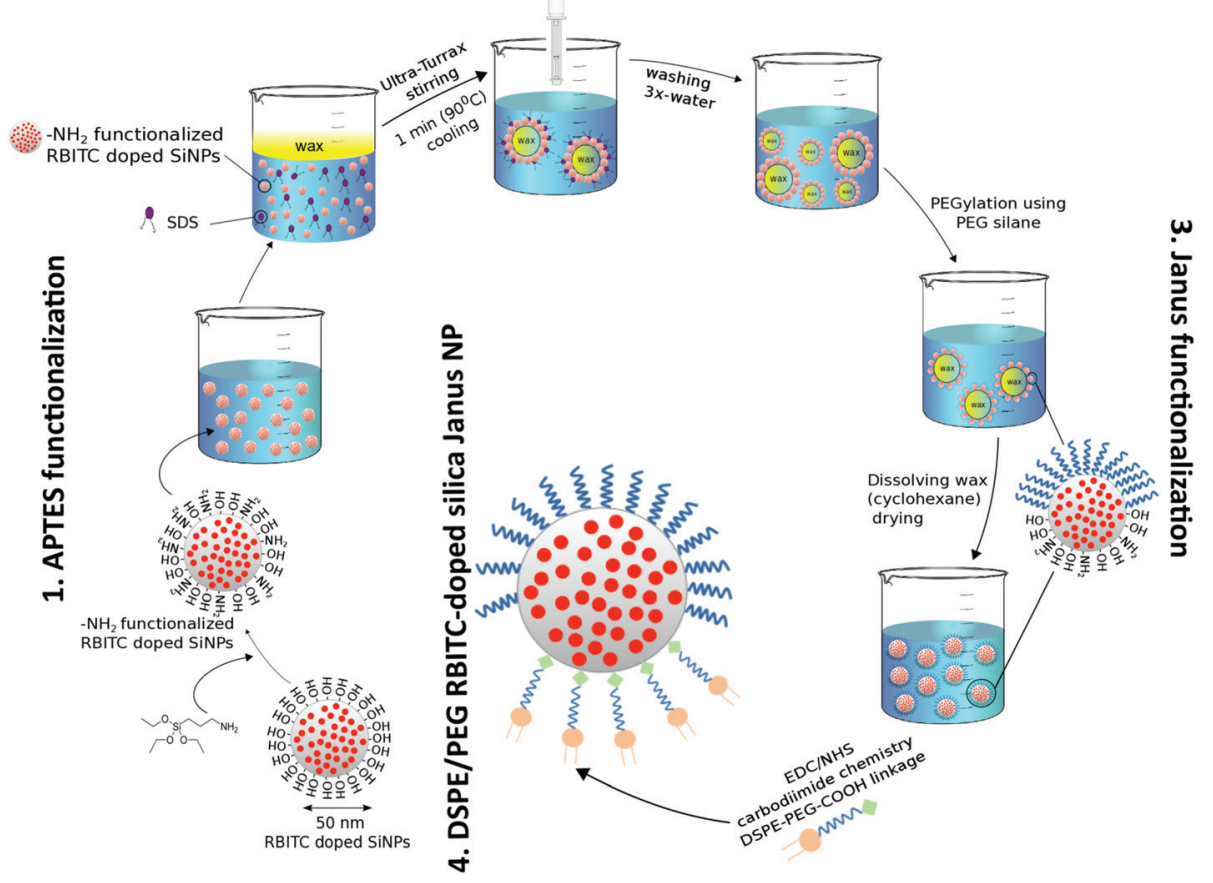

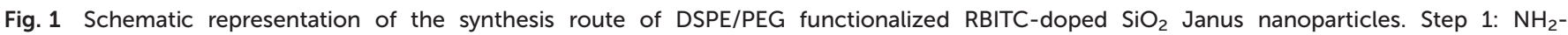
functionalization using silanization with APTES on $\mathrm{RBITC}$-doped $\mathrm{SiO}_{2}$ nanoparticles. Step 2: Wax Pickering emulsions are prepared with the $\mathrm{NH}_{2}-$ functionalized nanoparticles. Step 3: The solidified wax droplets are functionalized with PEG-silane. Step 4: DSPE-PEG-COOH is conjugated to the non-PEGylated half of the nanoparticles based on EDC-NHS carbodiimide linkage.

Table 1 Size and surface properties of the various functionalized $\mathrm{RBITC}$-doped $\mathrm{SiO}_{2}$ nanoparticles. Particle size, PDI and zeta potential analysis was measured in a $4 \%$ ethanol in water mixture and in full cell culture medium consisting of DMEM, FCS and AB/AM. Quantification of the $\mathrm{NH}_{2}$ groups was performed using the ninhydrin assay in water. The standard deviation is derived from three independent measurements

\begin{tabular}{|c|c|c|c|c|c|c|c|}
\hline & Liquid medium & Unfunctionalized & APTES & APTES/PEG & PEG & DSPE & DSPE/PEG \\
\hline & $\mathrm{DMEM}+\mathrm{FCS}+\mathrm{AB} / \mathrm{AM}$ & $52.4 \pm 7.3$ & $62.4 \pm 4.3$ & $67.2 \pm 2.3$ & $58.7 \pm 2.6$ & $86.9 \pm 7.8$ & $89.7 \pm 3.2$ \\
\hline PDI & $4 \%$ ethanol/water & $0.13 \pm 0.03$ & $0.15 \pm 0.04$ & $0.18 \pm 0.01$ & $0.14 \pm 0.09$ & $0.25 \pm 0.05$ & $0.21 \pm 0.03$ \\
\hline Zeta potential (mV) & $4 \%$ ethanol/water & $-31.4 \pm 4.4$ & $7.4 \pm 8.7$ & $-26.3 \pm 1.6$ & $-32.5 \pm 7.4$ & $-35.2 \pm 6.7$ & $-40.4 \pm 6.4$ \\
\hline & $\mathrm{DMEM}+\mathrm{FCS}+\mathrm{AB} / \mathrm{AM}$ & $-16.7 \pm 2.6$ & $-8.3 \pm 2.1$ & $-7.6 \pm 4.2$ & $-30.4 \pm 6.2$ & $-20.4 \pm 5.6$ & $-24.4 \pm 3.7$ \\
\hline $\mathrm{NH}_{2}$ groups/surface area $\left(\mathrm{nm}^{2}\right)$ & Water & $1.8 \pm 0.3$ & $20.7 \pm 6.8$ & $15.3 \pm 7.4$ & $4.3 \pm 1.8$ & $4.5 \pm 3.4$ & $2.5 \pm 1.6$ \\
\hline
\end{tabular}

amic diameter of PEGylated nanoparticles relative to that of unfunctionalized nanoparticles can in this case be attributed to the long-chain PEG groups functionalized on the $\mathrm{SiO}_{2}$ nanoparticles. The APTES/PEG Janus nanoparticles show somewhat decreased colloidal stability with higher hydrodynamic radius as a result of the anisotropic functionalization. Because of their increased hydrophobicity compared to the hydrophilic unfunctionalized and APTES-functionalized particles, fully DSPE-coated particles show again higher particle sizes and wider size distribution indicated by an increased polydispersity index (PDI) of 0.25 possibly due to agglomeration of the hydrophobic nanoparticles in the aqueous medium. Finally, the DSPE/PEG Janus nanoparticles displayed a hydrodynamic diameter of $86.2 \pm 10.4 \mathrm{~nm}$ and $89.7 \pm 3 \mathrm{~nm}$ in ethanol/water and cell culture medium, respectively (Table 1). Along this line, we most likely see some aggregation of DSPE/PEG Janus nanoparticles in the form of a mixture of dimeric agglomerates and individually dispersed particles possibly as a consequence of both the increased hydrophobicity of the DSPE and the Janus character of the particles. We expect that dimeric particles with the DSPE hemispheres pointing towards each other will behave similar to fully PEG-coated particles in subsequent experiments. Note that TEM analysis of the agglomeration state of the Janus particles is not suitable, since the drying process during sample preparation causes agglomeration, as well. The size of the DSPE/PEG nanoparticles seems to be unaffected by the presence of the otherwise surface-active molecules in the cell culture medium.

These findings are further substantiated by zeta potential measurements of all nanoparticle types in ethanol/water and 
cell culture medium, respectively (Table 1). Of special note is the positive surface charge of APTES-coated nanoparticles in ethanol/water which reverts to a negative charge in cell culture medium as a result of protein adsorption. ${ }^{32,37}$ Correspondingly, a decrease in zeta potential was observed for the APTES/PEG Janus nanoparticles resuspended in cell culture medium. In all other cases, regardless of the dispersion medium, the nanoparticles remained negatively charged.

Quantification of primary amino groups on the as-prepared nanoparticles was performed using the ninhydrin assay. Here, $20.7 \pm 6.8 \mathrm{NH}_{2}$ groups per $\mathrm{nm}^{2}$ of the surface of $\mathrm{NH}_{2}$-functionalized $\mathrm{SiO}_{2}$ nanoparticles were determined and Janus functionalization of the APTES/PEG nanoparticles reduced the number of quantifiable $\mathrm{NH}_{2}$ groups to $15.3 \pm 7.4$ amino groups per $\mathrm{nm}^{2}$ of the particle surface.

The success of the PEG-functionalization was further assessed by incubating the particles with FITC-BSA and analyzing the ratio of FITC-BSA adsorbed to the surface of nanoparticles to dissolved FITC-BSA in the supernatant (Fig. S2 $\dagger$ ). After $1 \mathrm{~h}$ of incubation, unfunctionalized and APTES-functionalized nanoparticles showed high amounts of FITC-BSA adsorption (Fig. S2A, $\uparrow$ black bars), whereas negligible amounts of BSA were detected in the respective supernatant (Fig. S2B, $\uparrow$ grey bars). On the other hand, APTES/PEG functionalized nanoparticles showed lower amounts of adsorbed FITC-BSA in comparison to the unfunctionalized and the positively charged APTES functionalized nanoparticles. As control, adsorption of FITC-BSA to PEG functionalized RBITC $\mathrm{SiO}_{2}$ nanoparticles was measured. Low amounts of FITC-BSA on the nanoparticles were detected confirming the success of PEG functionalization.

For FITC-BSA adsorption studies on both DSPE- and DSPE/ PEG functionalized particles, negligible amounts of FITC-BSA adsorption was observed on both particle types. The amounts of BSA adsorption are comparable to those obtained with fully PEG functionalized particles, showing that the DSPE-coating also prevents BSA adsorption.

Lastly, direct visualization of the immobilized organic molecules on the surface of the nanoparticles is extremely difficult using TEM due to their poor contrast. In a previous study, we grafted proteins bearing ultra-small gold or iron oxide nanoparticles on Janus particles to provide contrast in TEM, which more directly demonstrated the success of our approach. ${ }^{23}$ While the wax Pickering emulsion technique achieves the preparation of bulk quantities of Janus particles, we also observed that, with nanoparticles, the method does not provide a well-defined Janus balance. ${ }^{16,23}$

\section{CLSM analysis of nanoparticle interaction and uptake by cultured NIH 3T3 cells}

Confocal laser scanning microscopy (CLSM) was used to investigate the potential attachment and cellular uptake of nanoparticles by cultured NIH 3T3 cells. Fig. 2 shows representative CLSM images of cultured NIH 3T3 cells incubated with DSPEand DSPE/PEG-functionalized nanoparticles for $1 \mathrm{~h}, 6 \mathrm{~h}$ and

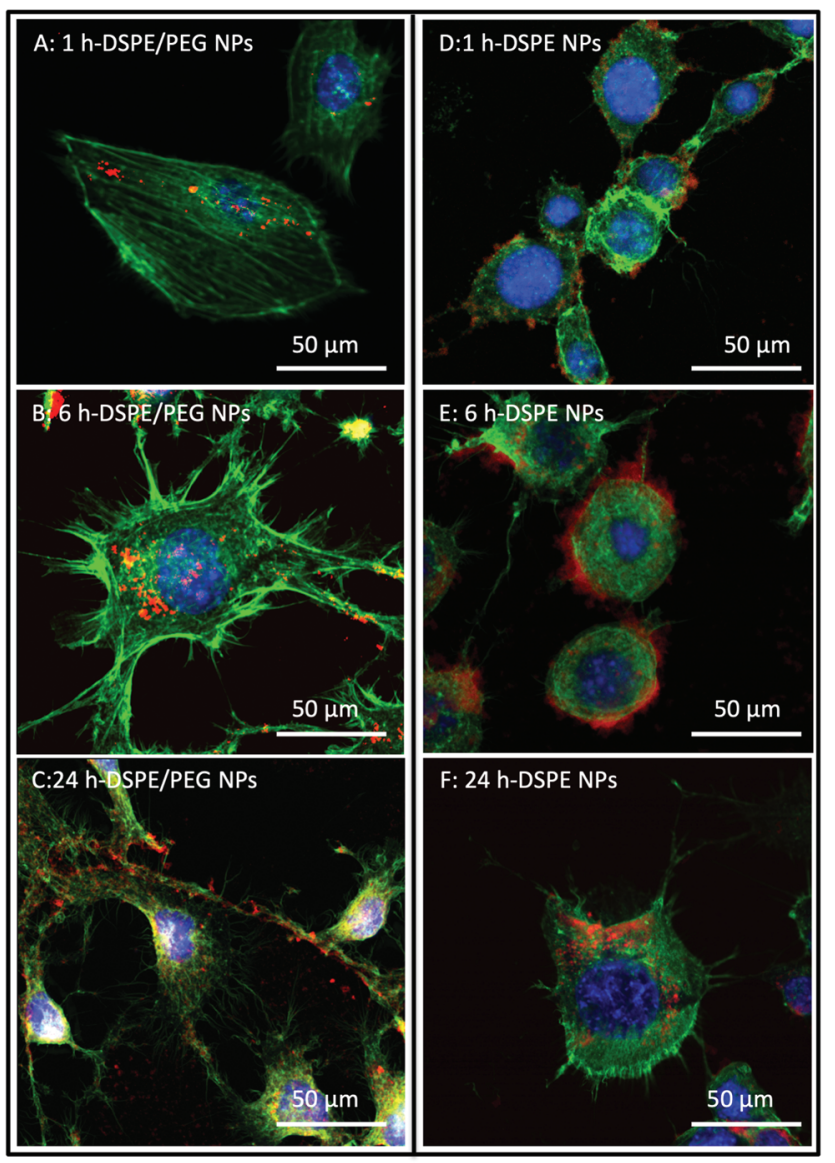

Fig. 2 CLSM images of NIH 3T3 cells after exposure to Janus DSPE/ PEG and fully DSPE functionalized nanoparticles. The cells were incubated with the nanoparticles for $1 \mathrm{~h}(\mathrm{~A}, \mathrm{D}), 6 \mathrm{~h}(\mathrm{~B}, \mathrm{E})$ and $24 \mathrm{~h}(\mathrm{C}, \mathrm{F})$ followed by fluorescence staining with specific fluorescent dyes. The nuclei and cytoskeletons are stained using the membrane impermeable dyes DAPI (blue) and phalloidin-AF-488 (green) respectively, whereas the nanoparticles are labelled with RBITC (red).

$24 \mathrm{~h}$. The micrographs revealed the association of the nanoparticles to the cells at all time points (Fig. 2A-F). Since the cells are very flat, it was not possible to unequivocally discern with $Z$-stack images whether the particles were localized inside the cytosol or whether they stayed attached to the surfaces of the cells (see Fig. $\mathrm{S} 3 \dagger$ for a series of $z$-stack images). As expected, control experiments without nanoparticles and PEGfunctionalized nanoparticles showed no particle association with the cells (Fig. S4†). In contrast, unfunctionalized and APTES-functionalized nanoparticles showed similar or, in the case of APTES, more frequent association relative to DSPE and DSPE/PEG functionalized nanoparticles (Fig. S5 $\dagger$ ). As mentioned above, APTES-functionalized particles undergo protein adsorption in cell culture medium causing a change in the surface charge. However, the serum proteins adsorbing onto the APTES-functionalized particles did not prevent uptake into cells, which is in accordance with the results published by Shahabi et al. ${ }^{33}$ 


\section{TEM analysis of particle uptake by cultured NIH 3T3 cells}

To further observe the cellular uptake of nanoparticles by cultured NIH 3T3 cells, TEM analysis was performed using $50 \mathrm{~nm}$ microtome sections of resin-embedded trypsinized cells after their exposure to nanoparticles at different time points (Fig. 3, S6 and S7 $\dagger$ ). Trypsin treatment detaches adherend cells from tissue culture plates and transfers them into suspension. In TEM analysis, such suspended spherically shaped cells allow efficient cellular localization of nanoparticles at the plasma membrane and in the cytosol. In the micrographs, the cytosol of the cells can be observed as darker gray areas (Fig. 3). Control experiments using untreated NIH 3T3 cells showed spherical features as lighter gray spots inside the cells that are most likely gas vacuoles (Fig. S7A-F $\dagger$ ). These gas vacuoles were also observed when cells were incubated with nanoparticles.
The nanoparticles themselves can be observed as dark gray dots (see the red arrows in Fig. 3) which fit to the DLS-diameter of the particles listed in Table 1.

When incubated with DSPE/PEG Janus nanoparticles, the nanoparticles remained constrained to the outer regions of the cells at all studied time points (Fig. 3A-F). Conversely, when cells were incubated with fully DSPE-functionalized nanoparticles, at $1 \mathrm{~h}$ (Fig. 3A and B), nanoparticle accumulation was observed on the cell surfaces. After $6 \mathrm{~h}$ of incubation (Fig. 3C and D), the nanoparticles were taken up into cellular microvesicles which was even more pronounced after $24 \mathrm{~h}$ of incubation (Fig. 3E and F). Most strikingly, a clear distinction between DSPE/PEG- and DSPE-functionalized nanoparticles was observed after $24 \mathrm{~h}$ of incubation. The majority of DSPEfunctionalized nanoparticles were localized inside the cells and, in contrast, when cells were incubated with DSPE/PEG
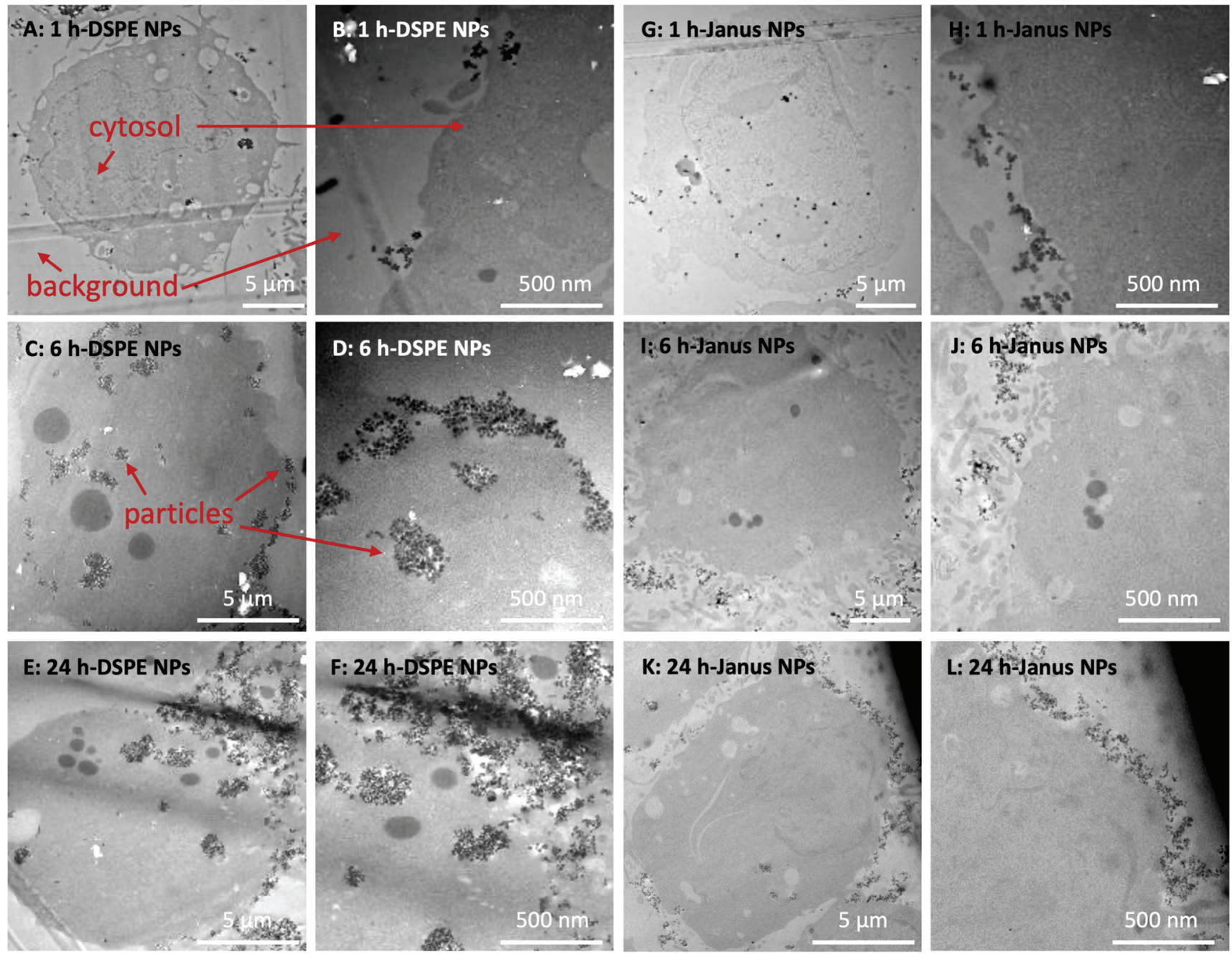

Fig. 3 TEM of the NIH 3 T3 cells after incubation with Janus DSPE/PEG- and isotropic DSPE-functionalized nanoparticles for $1 \mathrm{~h}, 6 \mathrm{~h}$ and $24 \mathrm{~h}$. The images of the second and forth column were taken at higher magnification as the images of the first and third column. Each sample was first embedded in epoxy resin followed by preparing ultrathin microtome sections of up to $50 \mathrm{~nm}$ thickness followed by TEM analysis. The red arrows indicate the main sources of contrast of the images from which all deductions are inferred. 
Janus particles, almost no internalization was observed (see Fig. S6 $†$ for further images with these particles). Additionally, the DSPE/PEG Janus nanoparticles seem to be mainly located at extracellular ruffled protrusions of the cell membrane which could possibly be associated to filipodia of the plasma membrane. Fig. S7 and S $\$ \uparrow$ illustrates the internalization of all other types (see Table 1) of synthesized nanoparticles which is clearly visible already after $1 \mathrm{~h}$ of incubation, as expected.

Particle uptake in the presence of endocytosis inhibitors

To further elucidate the endocytosis pathways that are potentially involved in the internalization of functionalized and unfunctionalized nanoparticles, cultured NIH 3T3 cells were incubated with our synthesized nanoparticles in the presence or absence of the common endocytosis inhibitors nystatin and wortmannin as well as with high-sucrose medium and at $4{ }^{\circ} \mathrm{C}$. Fig. 4 shows representative fluorescence microscopy images of NIH 3T3 cells incubated for $6 \mathrm{~h}$ with the respective nanoparticles under the mentioned conditions. Additionally, the results for $1 \mathrm{~h}$ and $24 \mathrm{~h}$ of incubation are shown in Fig. S9 and S10.† Cell nuclei are stained with DAPI and are illustrated in blue and the RBITCdoped $\mathrm{SiO}_{2}$ nanoparticles are shown in red. Green actin staining has not been included in these images for clarity but is included in ESI Fig. S11-S13† as reference.

As expected, without nanoparticles and with fully PEGcoated particles, no particles are visible in any experiments

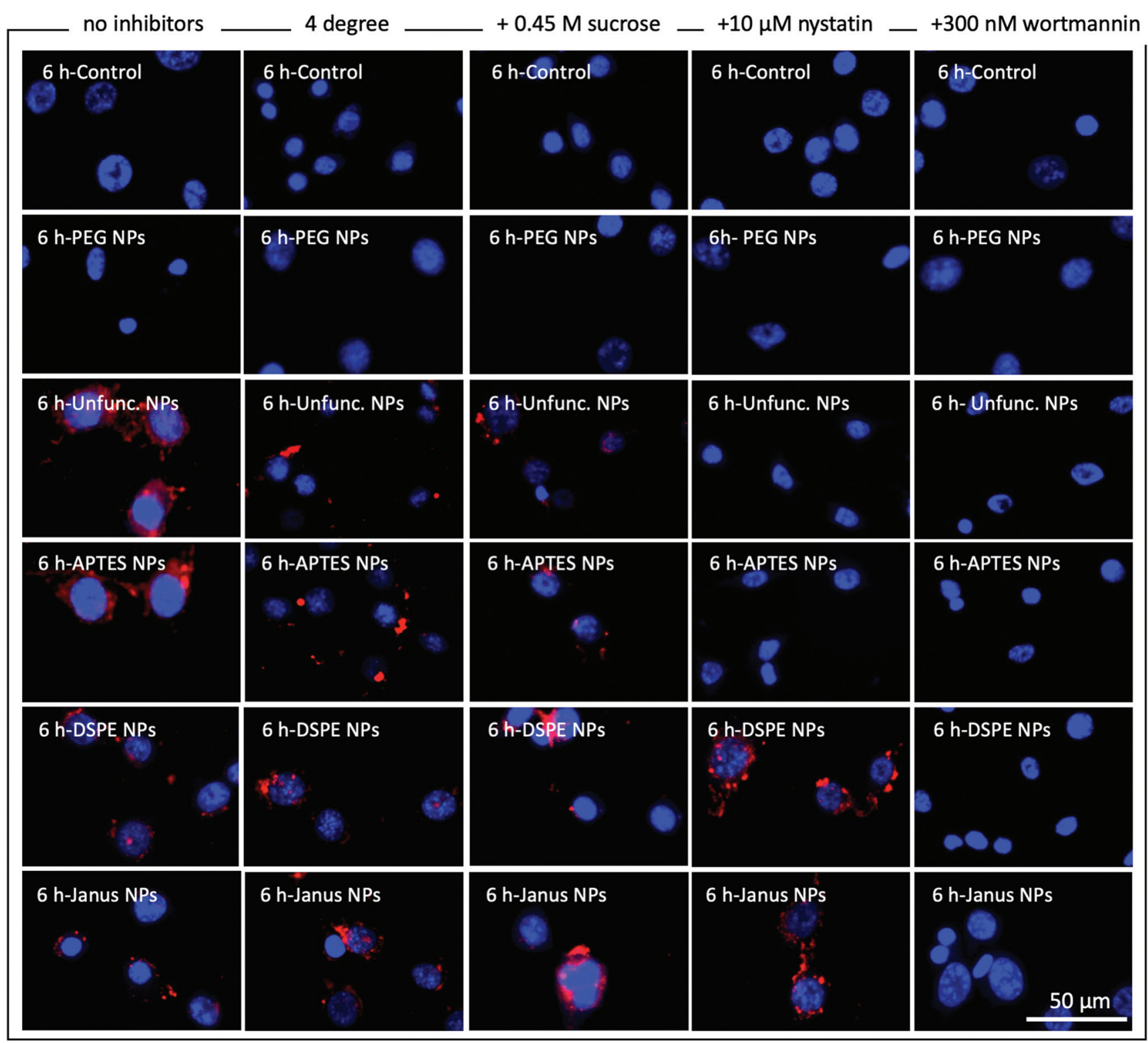

Fig. 4 Fluorescence microscopy of NIH 3T3 cells before (control) and after exposure of the different nanoparticles for $6 \mathrm{~h}$ in the presence and absence of endocytosis inhibitors. $4{ }^{\circ} \mathrm{C}$ incubation was performed to suppress temperature-dependent endocytosis, hypertonic sucrose concentrations suppressed receptor-mediated endocytosis, nystatin inhibits caveolae-mediated endocytosis and wortmannin inhibits macropinocytosis. The nuclei were stained using DAPI (blue), whereas the nanoparticles were labelled with RBITC (red). 
(Fig. 4, first two rows). As discussed above, unfunctionalized and APTES-coated nanoparticles were strongly internalized in the absence of endocytosis inhibitors (Fig. 4, third row, see also Fig. S5 and S8†). In direct comparison, DSPE- and DSPE/ PEG-functionalized nanoparticles seem to be taken up to a lesser degree than APTES-coated and unfunctionalized nanoparticles (Fig. 4, fourth and fifth row). However, on the basis of the fluorescent microscopy images it is difficult to make a distinction between surface-attached and internalized nanoparticles. We assume that all visible extracellular nanoparticles are somehow associated to the cell surface since these still remained after the washing steps. Temperature-dependent suppression of endocytosis by incubating NIH $3 \mathrm{~T} 3$ cells at $4{ }^{\circ} \mathrm{C}$ (Fig. 4, second column) caused a strong decrease or slowdown in the uptake of the unfunctionalized and APTES-functionalized particles. This mitigation of uptake was also detected for DSPE- and DSPE/PEG-functionalized nanoparticles after $1 \mathrm{~h}$ of incubation (Fig. S9†), but is no longer visible after $6 \mathrm{~h}$ and $24 \mathrm{~h}$ (Fig. 4 and Fig. S10†). Incubation of NIH 3T3 cells in hypertonic media containing $0.45 \mathrm{M}$ sucrose should overload cell surface receptors involved in receptor-mediated endocytosis (Fig. 4, third column). ${ }^{38}$ This treatment also resulted in lower uptake rates of unfunctionalized and APTES-functionalized nanoparticles relative to those observed for untreated samples incubated under standard culturing conditions (Fig. 4, first column). However, cellular uptake of DSPE- and DSPE/PEG-functionalized nanoparticles remained unaffected by the high-sucrose medium treatment. The same trend can be observed when the endocytosis inhibitor nystatin is added to the cell culture medium (Fig. 4, fourth column). Nystatin is known to inhibit caveola-dependent endocytosis and seems to be exclusively effective to prevent cellular uptake of the hydrophilic unfunctionalized and APTES-functionalized nanoparticles. Along this line, in the presence of wortmannin (Fig. 4, fifth column), a well-characterized macropinocytosis inhibitor, no intracellular accumulation was observed for any nanoparticle type.

While a straightforward interpretation of these results is difficult since several endocytosis pathways might be active for one particle type, they indicate that both caveolae-dependent endocytosis and macropinocytosis are involved in the uptake of isotropic APTES-coated and unfunctionalized nanoparticles. The interaction of NIH 3T3 cells with isotropic DSPE- and DSPE/PEG-functionalized Janus nanoparticles is not strongly affected by the presence of nystatin or the high sucrose concentration. Conversely, suppression of macropinocytosis via wortmannin inhibited the association of both DSPE- and DSPE/PEG-functionalized nanoparticles. These results indicate that macropinocytosis seems to be the main endocytosis pathway for the uptake of the fully DSPE-coated nanoparticles. Although the TEM analysis (Fig. 3) suggests that the DSPE/ PEG-functionalized Janus nanoparticles are mainly attached to the cell surface and are only barely taken up to some extent, some effects of macropinocytosis seem to be involved in firmly attaching the nanoparticles to the cell surface. This is to some degree substantiated by the observation of the roughened cell surface in the presence of the Janus nanoparticles in the TEM micrographs (Fig. 3A-F). These results could be an evidence for an interrupted macropinocytosis process that accumulates the nanoparticles at the cell surface without forming closed macropinosomes and subsequent transfer into the cytosol.
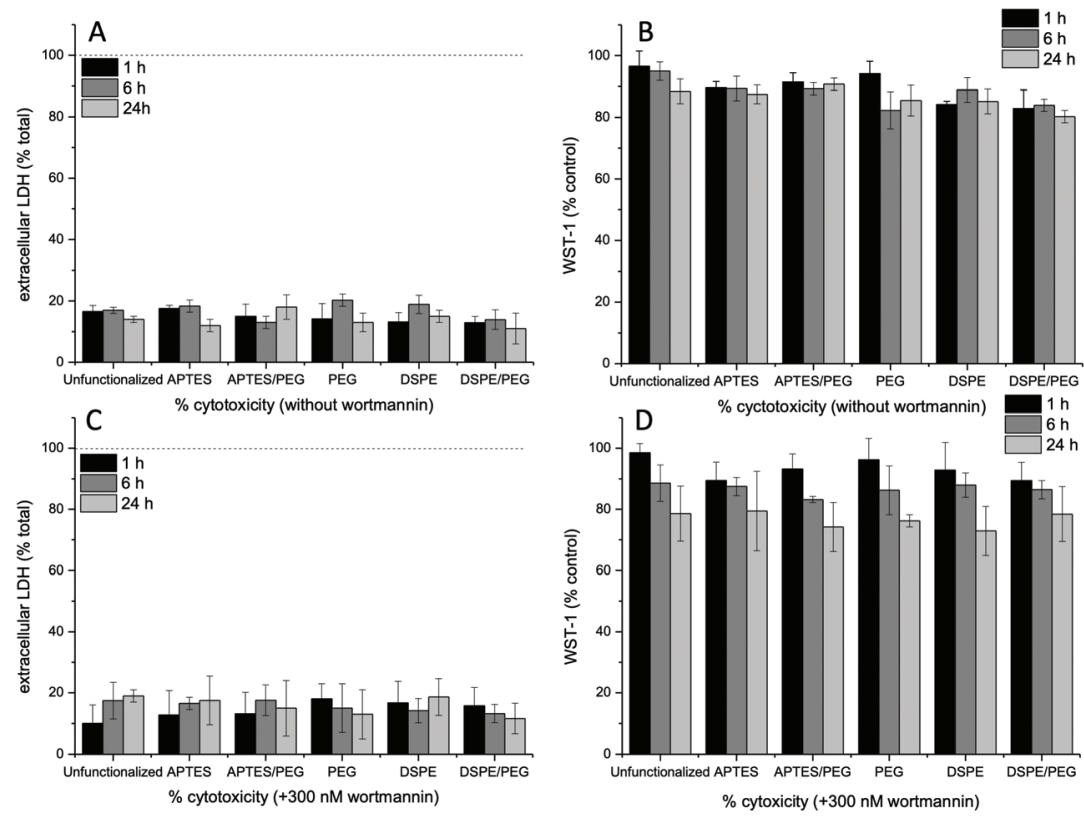

Fig. 5 Cell viability analysis of NIH 3T3 cells after incubation with the different nanoparticle types in the presence and absence of the macropinocytosis inhibitor wortmannin for 1, 6 and $24 \mathrm{~h}$ of incubation. Membrane integrity was measured via the LDH assay (A, C) whereas mitochondrial viability was assessed with the WST-1 assay (B, D). 


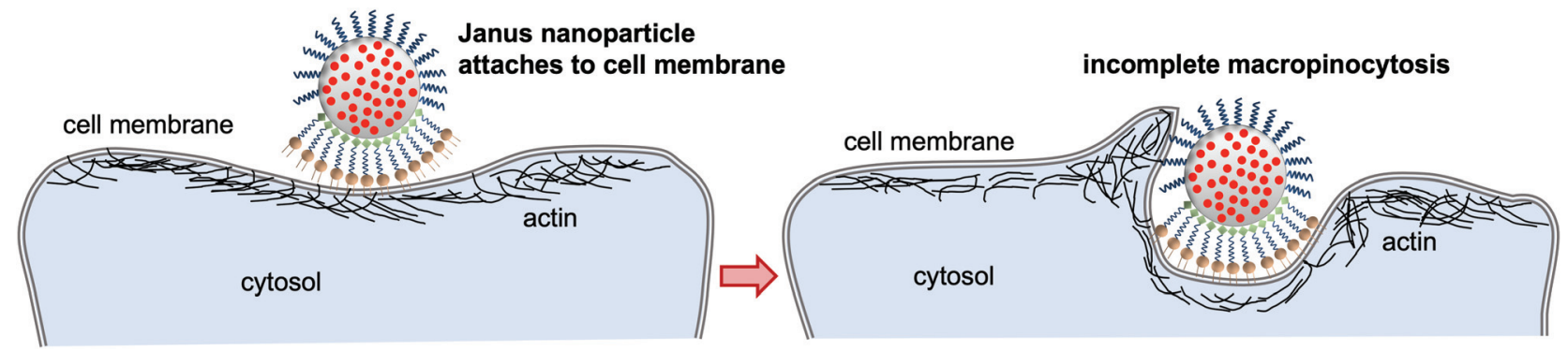

Fig. 6 Scheme of the assumed interactions of the Janus nanoparticles with the cell membrane. The particles attach to the membrane via insertion of the hydrophobic tails of the DSPE functionalization in the phospholipid bilayer of the cell membrane. This triggers macropinocytosis which is obstructed by the PEG functionalization leading to firm attachment of the particle to the cell surface without full endocytosis.

\section{Cell viability}

As discussed above, in the presence of endocytosis inhibitors, nanoparticle uptake and/or interaction with cell surface molecules is affected. Hence, the viability of the cells in the presence of the inhibitors in combination with the different nanoparticle types was analyzed. To this end, we used the WST-1 cell proliferation assay and quantified the release of cytosolic LDH, which indicates membrane and consequently cell damage, and thus can be used to determine cell viability. In the absence of endocytosis inhibitors, overall, LDH release of around $20 \%$ and a slight reduction of cellular WST-1 was observed (Fig. 5A and B). The LDH release was generally unaffected by the type of nanoparticle used, regardless of the addition of inhibitors. Without wortmannin at $24 \mathrm{~h}$, a slight decrease of the WST-1 reduction capacity (85\%) was recorded in the presence of DSPE/PEG-functionalized Janus nanoparticles. This might be a result of the observed deformations of the cell surface and the associated stresses to the cells. The presence of wortmannin slightly affects mitochondrial activity, as observed by a WST- 1 reduction capacity to $75-80 \%$ at $24 \mathrm{~h}$ of incubation. Similar observations were made in the presence of the other endocytosis inhibitors sucrose (Fig. S14A and $\mathrm{C}^{\dagger}$ ) and nystatin (Fig. S14B and $\mathrm{D} \dagger$ ). Here, WST-1 reduction capacity was decreased in the presence of nystatin (80\%) and high-sucrose media (70\%) with all nanoparticle types at the $24 \mathrm{~h}$ time point. Since further degradation in cell viability would make interpretation of the experiments difficult, we did not perform longer incubation experiments.

\section{Conclusions}

In summary, we synthesized DSPE/PEG-functionalized Janus nanoparticles, along with a series of non-Janus nanoparticles, via the wax-in-water Pickering emulsion strategy. All types of nanoparticles showed reasonable colloidal stability, particularly in the cell medium in which the endocytosis experiments were carried out. The isotropic nanoparticles were taken up into the cells with the exception of the fully PEG-coated nanoparticles. This uptake behavior was expected and is also welldocumented in the literature. ${ }^{1,2}$ Conversely, the DSPE/PEG- functionalized Janus nanoparticles behave similarly to the Janus microparticles investigated by Gao and $\mathrm{Yu}^{7}$ which generate relatively large membrane protrusions that eventually engulf the microparticles via the macropinocytosis pathway. Surprisingly, the nano-sized Janus particles investigated in our study are not taken up at all and only seem to be grabbed by the cell membrane in a process that can be inhibited by the presence of wortmannin during incubation (schematically shown in Fig. 6). Accordingly, we assume that an incomplete macropinocytosis process might be responsible for accumulating the Janus nanoparticles at the cell surface. Further studies are necessary to transfer these findings to other cell types. It will also be interesting to analyze in a further study whether the hydrophobic membrane anchor might in part be responsible for the observations, compared, e.g., to particles binding to a CD3 receptor.

Finally, anisotropic particles that are designed to stay permanently attached to membrane surfaces might prove eminently useful in biomedical applications. Particularly magnetized Janus nanoparticles could be able to move or otherwise manipulate individual cells non-invasively by serving as a magnetic handle at the cell membrane leading to novel tools for the field of magnetic tissue engineering. ${ }^{39}$ Furthermore, nature has already brought forth anisotropic nanoparticles that are able to inject their DNA or RNA into cells after latching onto cell surfaces. Accordingly, myovirus bacteriophages and similar viruses could act as templates for even more sophisticated anisotropic nanoparticle drug or gene delivery systems in the future.

\section{Conflicts of interest}

There are no conflicts to declare.

\section{References}

1 N. Oh and J.-H. Park, Int. J. Nanomed., 2014, 9, 51-63.

2 S. Zhang, H. Gao and G. Bao, ACS Nano, 2015, 9, 86558671. 
3 R. Vácha, F. J. Martinez-Veracoechea and D. Frenkel, Nano Lett., 2011, 11, 5391-5395.

4 S. Schöttler, G. Becker, S. Winzen, T. Steinbach, K. Mohr, K. Landfester, V. Mailänder and F. R. Wurm, Nat. Nanotechnol., 2016, 11, 372-377.

5 L. Sanchez, Y. Yi and Y. Yu, Nanoscale, 2017, 9, 288-297.

6 L. Treccani, T. Yvonne Klein, F. Meder, K. Pardun and K. Rezwan, Acta Biomater., 2013, 9, 7115-7150.

7 Y. Gao and Y. Yu, J. Am. Chem. Soc., 2013, 135, 1909119094.

8 P.-G. de Gennes, Angew. Chem., Int. Ed. Engl., 1992, 31, 842845.

9 Y. Yi, L. Sanchez, Y. Gao and Y. Yu, Analyst, 2016, 141, 3526-3539.

10 D. Shao, J. Li, X. Zheng, Y. Pan, Z. Wang, M. Zhang, Q.-X. Chen, W.-F. Dong and L. Chen, Biomaterials, 2016, 100, 118-133.

11 L. Y. Wu, B. M. Ross, S. Hong and L. P. Lee, Small, 2010, 6, 503-507.

12 F. Wang, G. M. Pauletti, J. Wang, J. Zhang, R. C. Ewing, Y. Wang and D. Shi, Adv. Mater., 2013, 25, 3485-3489.

13 W. He, J. Frueh, Z. Wu and Q. He, Langmuir, 2016, 32, 3637-3644.

$14 \mathrm{~W}$. He, J. Frueh, Z. Wu and Q. He, ACS Appl. Mater. Interfaces, 2016, 8, 4407-4415.

15 Z. Wang, Y. Wang, Z. Chang, L. Li, Y. Zhang, M. Lu, X. Zheng, M. Li, D. Shao, J. Li, L. Chen and W. Dong, Chem. Biol. Drug Des., 2017, 89, 464-469.

16 R. Kadam, M. Maas and K. Rezwan, ACS Appl. Bio Mater., 2019, 2, 3520-3531.

17 Z. Chang, Z. Wang, M. Lu, M. Li, L. Li, Y. Zhang, D. Shao and W. Dong, RSC Adv., 2017, 7, 3550-3553.

18 E. Poggi and J.-F. Gohy, Colloid Polym. Sci., 2017, 295, 2083-2108.

19 C. Kaewsaneha, P. Tangboriboonrat, D. Polpanich, M. Eissa and A. Elaissari, Colloids Surf., A, 2013, 439, 35-42.

20 X. Y. Ling, I. Y. Phang, C. Acikgoz, M. D. Yilmaz, M. A. Hempenius, G. J. Vancso and J. Huskens, Angew. Chem., Int. Ed., 2009, 48, 7677-7682.
21 L. Hong, S. Jiang and S. Granick, Langmuir, 2006, 22, 94959499.

22 J. Zhang, B. A. Grzybowski and S. Granick, Langmuir, 2017, 33, 6964-6977.

23 R. Kadam, M. Zilli, M. Maas and K. Rezwan, Part. Part. Syst. Charact., 2018, 35, 1700332.

24 Y. Gao and Y. Yu, Langmuir, 2015, 31, 2833-2838.

25 C. A. S. Batista, R. G. Larson and N. A. Kotov, Science, 2015, 350, 1242477.

26 H. Ding and Y. Ma, Small, 2015, 11, 1055-1071.

27 T. Bollhorst, S. Shahabi, K. Wörz, C. Petters, R. Dringen, M. Maas and K. Rezwan, Angew. Chem., 2015, 127, 120-125.

28 R. Watanabe, T. Yokoi, E. Kobayashi, Y. Otsuka, A. Shimojima, T. Okubo and T. Tatsumi, J. Colloid Interface Sci., 2011, 360, 1-7.

29 E. Soto-Cantu, R. Cueto, J. Koch and P. S. Russo, Langmuir, 2012, 28, 5562-5569.

30 D. Li, Q. He, Y. Cui, L. Duan and J. Li, Biochem. Biophys. Res. Commun., 2007, 355, 488-493.

31 H. D. Chirra, T. Sexton, D. Biswal, L. B. Hersh and J. Z. Hilt, Acta Biomater., 2011, 7, 2865-2872.

32 S. Shahabi, S. Döscher, T. Bollhorst, L. Treccani, M. Maas, R. Dringen and K. Rezwan, ACS Appl. Mater. Interfaces, 2015, 7, 26880-26891.

33 S. Shahabi, L. Treccani, R. Dringen and K. Rezwan, ACS Appl. Mater. Interfaces, 2015, 7, 13821-13833.

34 M. G. Holthaus, J. Stolle, L. Treccani and K. Rezwan, Acta Biomater., 2012, 8, 394-403.

35 K. Apte, R. Stick and M. Radmacher, J. Mol. Recognit., 2017, 30, e2580.

36 V. Lauth, M. Maas and K. Rezwan, Mater. Sci. Eng., C, 2017, 78, 305-314.

37 F. Meder, T. Daberkow, L. Treccani, M. Wilhelm, M. Schowalter, A. Rosenauer, L. Mädler and K. Rezwan, Acta Biomater., 2012, 8, 1221-1229.

38 J. E. Heuser and R. G. Anderson, J. Cell Biol., 1989, 108, 389-400.

39 J. R. Henstock, H. Markides, H. Bin, A. J. E. Haj, J. Dobson, H. Markides, H. Bin, A. J. E. Haj and J. Dobson, in Nanomagnetic Actuation in Biomedicine, 2018. 